\title{
A filosofia da Medicina de Família e Comunidade segundo lan McWhinney e Roger Neighbour
}

The philosophy of Family and Community Medicine according to lan McWhinney and Roger Neighbour

\section{La filosofía de la Medicina Familiar y Comunitaria según lan McWhinney y Roger Neighbour}

\author{
Beatriz Ramirez Jaco ${ }^{1} \mathbb{D}$, Armando Henrique Norman² ${ }^{\mathbb{D}}$ \\ ${ }^{1}$ Escola de Saúde Pública da Secretaria Municipal de Saúde de Florianópolis, Florianópolis, Santa Catarina, Brasil \\ ${ }^{2}$ Universidade Federal de Santa Catarina, Florianópolis, Santa Catarina, Brasil
}

\section{Resumo}

Introdução: O presente artigo revisita os pressupostos filosóficos da Medicina de Família e Comunidade (MFC) a partir dos escritos de lan McWhinney e Roger Neighbour. Objetivo: Fortalecer a discussão sobre as bases teóricas da MFC na academia e nos programas de residência em MFC. Métodos: Trata-se de um ensaio reflexivo que compara e analisa dois dos principais livros da MFC: o clássico "Manual de Medicina de Família" de lan McWhinney e "The inner physician: why and how to practise 'Big Picture Medicine"' de Roger Neighbour. Resultados e Discussão: lan McWhinney e Roger Neighbour utilizam a epistemologia de Thomas Kuhn para propor um paradigma diferente à medicina. Nesse processo, os autores desenvolveram propostas distintas, porém complementares, que optamos por categorizar em: (a) paradigma organísmico de McWhinney e quântico de Neighbour; (b) distinção entre a prática generalista e a do especialista focal; e (c) relação médico-paciente e consigo mesmo. Conclusão: Para navegar nas incertezas da prática generalista é necessário fomentar e refletir a respeito da essência do MFC, tanto nos cursos de graduação quanto nos programas de residência, para formar profissionais sensíveis à condição humana.

Palavras-chave: Atenção Primária à Saúde; Filosofia; Medicina de Família e Comunidade; Internato e Residência; Educação Médica.

\begin{abstract}
Introduction:This article revisits the philosophical assumptions of Family and Community Medicine (FCM) from lan McWhinney's and Roger Neighbour's writings. Objective: To strengthen the discussion on the theoretical foundations of FCM both in the academy and in residency programmes in FCM. Methods: This is a reflexive essay that compares and analyses two of the main books on FCM: the classic "Textbook of Family Medicine" by lan McWhinney and "The inner physician: why and how to practise "Big Picture Medicine"' by Roger Neighbour. Results and Discussion: lan McWhinney and Roger Neighbour apply the epistemology of Thomas Kuhn to propose a different paradigm to medicine. In this process, the authors developed distinct but complementary proposals that we decided to categorise into: (a) McWhinney's organismic paradigm and Neighbour's quantum paradigm; (b) a distinction between general practitioner and focal specialist; and (c) doctor-patient relationship and with itself. Conclusion:To navigate the uncertainties of general practice, it is necessary to nurture and reflect on the essence of FCM, both in undergraduate courses and in residency programmes, to train professionals sensitive to the human condition.
\end{abstract}

Keywords: Primary Health Care; Philosophy; Family and Community Medicine; Internship and Residency; Medical Education.

Como citar: Jaco BR, Norman AH. A filosofia da Medicina de Família e Comunidade segundo Ian McWhinney e Roger Neighbour. Rev Bras Med Fam Comunidade. 2020;15(42):1991. https://doi.org/10.5712/rbmfc15(42)1991

\author{
Autor correspondente: \\ Beatriz Ramirez Jaco. \\ E-mail: be.ramirezj@gmail.com \\ Fonte de financiamento: \\ declaram não haver. \\ Parecer CEP: \\ não se aplica. \\ Procedência e revisão por pares: \\ revisado por pares. \\ Recebido em: 21/02/2019 \\ Aprovado em: 13/01/2020
}




\section{Resumen}

Introducción: El presente artículo revisita las premisas filosóficas de la Medicina de Familia y Comunidad (MFC) a partir de los escritos de lan McWhinney y Roger Neighbour. Objetivo: Fortalecer la discusión sobre las bases teóricas de la MFC en la academia y en los programas de residencia en MFC. Métodos: Se trata de un ensayo reflexivo que compara y analiza dos de los principales libros de la MFC: el clásico "Manual de Medicina de Familia" por lan McWhinney y "The inner physician: why and how to practise 'Big Picture Medicine"' por Roger Neighbour. Resultados y Discusión: lan McWhinney y Roger Neighbour utilizan la epistemología de Thomas Kuhn para proponer un paradigma diferente a la medicina. En ese proceso, los autores desarrollaran propuestas distintas, pero complementarias que optamos por categorizar en: (a) paradigma organísmico de McWhinney y cuántico de Neighbour; (b) distinción entre la práctica generalista y la del especialista focal; y (c) relación médico-paciente y consigo mismo. Conclusión: Para navegar en las incertidumbres de la práctica generalista es necesario fomentar y reflexionar sobre la esencia del MFC, tanto en los cursos de graduación como en los programas de residencia, para formar profesionales sensibles a la condición humana.

Palabras clave: Atención Primaria de Salud; Filosofía; Medicina de Familia y Comunidad; Internado y Residencia; Educación Médica.

\section{INTRODUÇÃO}

A Medicina de Família e Comunidade (MFC) é uma especialidade médica que surgiu para questionar o pensamento cientificista do século $X X$, em reação à tendência a superespecialização médica e a consequente precarização da relação médico-paciente. ${ }^{1}$ A prática do MFC tem seu foco na Atenção Primária à Saúde (APS) e os sistemas públicos de saúde estruturados a partir da APS têm papel importante no fomento dessa especialidade. ${ }^{2} \mathrm{Na}$ APS, os MFCs têm a função filtro (gatekeeper), visto que são a porta de entrada do sistema (primeiro contato). ${ }^{3}$ Nessa estrutura, o MFC cuida da saúde de seus pacientes de forma longitudinal, integral e coordenada; considerando seu contexto familiar e comunitário. Deste modo, o MFC precisa ter um olhar para dentro de si, do paciente e do contexto sociocultural para buscar extrair o sentido do encontro com aquele que procura por atendimento nos serviços de saúde. ${ }^{4}$

Da metade do século XX em diante, houve uma ressignificação, evolução e crescimento da MFC em âmbito global e muitos autores buscaram debater a complexidade da medicina de família, tanto em seus aspectos filosóficos, quanto em seus aspectos práticos. ${ }^{1}$

Este artigo aborda duas obras importantes da MFC, escritas por consagrados autores e médicos de família: lan McWhinney e Roger Neighbour. O primeiro é um dos mais respeitados médicos de família e comunidade no mundo. Recebeu diversos prêmios, publicou muitos artigos e livros no campo da MFC. Nascido na Inglaterra, onde estudou e cursou medicina e posteriormente se especializou como médico de família. Mudou-se para o Canadá, onde escreveu o livro “A Textbook of Family Medicine”, entre 1968 e 1987, enquanto era diretor do recémcriado departamento de medicina de família da Universidade de Western Ontário. Durante sua carreira explorou os conceitos do médico de família e seu paciente, influenciou a ciência e a medicina, mais especificamente a MFC. ${ }^{5} \mathrm{O}$ segundo autor é ainda pouco conhecido no Brasil, mas vem se destacando mundialmente no campo da MFC. Neighbour fez faculdade de medicina no King's College, em Cambridge, onde trabalhou como General Practitioner (GP). Seu interesse pela psicologia, pela relação médico-paciente e pela relação professor-aluno, levou-o a escrever a trilogia: "The Inner Consultation" (1987), " "The Inner Apprentice" (1992)7 e "The Inner Physician" (2016). ${ }^{8}$ Em 2003, foi eleito presidente do Royal College of General Practitioners por três anos.

Tradicionalmente, as escolas médicas no Brasil não dedicam tempo para a discussão sobre as bases epistemológicas da medicina. Essa ausência de um olhar crítico sobre sua práxis perpetua conceitos e gera verdades ilusórias. Para McWhinney e Freeman ${ }^{1}$ foi por meio da "fermentação de ideias" e de reflexões sobre esse modelo médico que a MFC atingiu um novo patamar e status enquanto especialidade médica. 
Este artigo revisita os pressupostos filosóficos da MFC a partir de McWhinney e Neighbour. Pretende-se fazer uma análise conceitual e filosófica, discutindo semelhanças e diferenças entre os dois autores e fomentando reflexões sobre a MFC. O objetivo é fortalecer a discussão na academia e nos programas de residência médica sobre as bases que alicerçam a MFC enquanto disciplina acadêmica.

\section{MÉTODOS}

Este estudo é um ensaio teórico que visa comparar os textos contidos em dois livros relevantes para a MFC: "Manual de Medicina de Família e Comunidade" (com tradução para o português) ${ }^{1}$ e "The inner physician: why and how to practise 'Big Picture Medicine'”, ainda sem tradução para o português. O primeiro é de autoria de lan McWhinney (1997) ${ }^{9}$ - embora na edição mais recente tenha a coautoria de Thomas Freeman (2010), ${ }^{1}$ os conceitos contidos já haviam sido elaborados pelo primeiro autor - e o segundo de Roger Neighbour.

\section{Critérios de inclusão e seleção dos capítulos}

Após a leitura dos livros, os autores "BRJ" e "AHN" avaliaram os capítulos que seriam incluídos para análise e posterior reflexão. Foram selecionados os seis primeiros capítulos do livro de McWhinney que estão contidos na primeira parte e têm como subtítulo os princípios básicos da Medicina de Família e Comunidade. Nesses primeiros capítulos McWhinney explora detalhadamente os pressupostos da MFC, foco do presente estudo. Os demais capítulos foram excluídos por terem conteúdos conceituais sobre a clínica e outros assuntos que não são o eixo deste ensaio.

O mesmo ocorreu com o livro de Roger Neighbour. Foram escolhidos os seis primeiros capítulos, visto que os cinco primeiros trazem as reflexões do autor sobre as bases da especialidade. No sexto capítulo, o autor resume os principais conceitos sobre a MFC. Em relação à comunicação clínica, área em que o autor é um expert, o sexto capítulo é um "summarising". Assim, para o escopo do estudo, esses capítulos foram suficientes para expressar a epistemologia de Neighbour sobre a MFC. Os demais capítulos são desenvolvimentos das ideias contidas nos primeiros cinco capítulos.

\section{Análise e tratamento dos textos}

A análise dos textos foi realizada de forma independente, por meio de uma reflexão crítica do conteúdo de cada capítulo. Foram selecionados os trechos que exprimiam os aspectos filosóficos que definem a especialidade da MFC. Os trechos foram então comparados e, por meio da discussão iterativa, em que um trecho selecionado leva a uma nova elaboração e a um refinamento de cada um deles, de modo a estruturar os temas a serem selecionados para a discussão e reflexão sobre as bases filosóficas da MFC.

\section{Perfil dos autores}

Como se trata de um estudo qualitativo com alto grau de subjetividade, é importante a reflexão sobre o perfil dos autores, ambos MFCs. "BRJ" é recém pós-graduada em MFC pelo programa de residência médica da Secretaria Municipal de Saúde de Florianópolis-SC. Seu olhar entusiástico pela especialidade e pouco tempo de vivência na prática profissional contribuíram para a seleção dos textos com uma visão menos influenciada pelos vieses da especialidade. 
"AHN", MFC experiente e preceptor desde 2001 de programas de residência médica em MFC trouxe um olhar mais acadêmico e da vivência profissional na condução da discussão sobre os textos, na seleção e análise dos capítulos. Essa combinação de diferentes olhares e experiências contribuiu para a riqueza dos textos selecionados.

\section{RESULTADOS E DISCUSSÃO}

Uma primeira característica que separa McWhinney de Neighbour é o momento em que cada um escreve seus livros. Finalizado na década de 1980, o livro-texto de McWhinney é um marco em termos de didatismo e organização das bases da MFC e, por isso, tem uma formalidade impecável. Neighbour escreve em uma época em que a MFC já se encontra bem estruturada no Reino Unido. Sua linguagem é mais coloquial, contemporânea e parte da perspectiva do olhar do médico de família. Nessa perspectiva, Neighbour toma como referência o que ele chama do "amador interno" ou "pessoa leiga" que vive nas profundezas dos médicos. Não obstante a linguagem mais informal, Neighbour consegue abordar com profundidade temas caros à MFC. Por exemplo, McWhinney explica pormenorizadamente o pensamento sistêmico e inclui uma figura para mostrar a complexidade dos vários níveis de interação. Por sua vez, Neighbour apresenta seu pensamento hierárquico e complexo por meio de uma matrioska, um conjunto de bonecas russas em que a maior boneca abriga dentro de si várias outras bonecas. Ele relaciona cinco níveis a partir da boneca mais externa até a mais interna: (1) Sistema de saúde e arena política; (2) O construto imaginário que a sociedade tem sobre o médico; (3) O especialista focal; (4) O médico de família e comunidade; e (5) O amador interno.

Neighbour (p. 10) $)^{8}$ utiliza a metáfora da matrioska com base no teórico húngaro Arthur Koester que "postula que cada nível hierárquico de organização tem objetivos impostos pelos níveis hierarquicamente superiores, mas cada nível é livre para desenvolver o modo como irá atingir suas metas". Já McWhinney (p. 93) 1 utiliza a Filosofia Perene e a do matemático Alfred Whitehead que afirma que há uma "hierarquia no nível existencial: o transcendente, o mental e o físico [...] cada nível mais alto tem capacidades não encontradas nos mais baixos, o mais alto não pode derivar dos mais baixos". Portanto, ambos se apoiam nas teorias sistêmicas, complexas e do caos em uma antítese ao reducionismo, nas quais "sistemas complexos exibem propriedades que não poderiam ser preditas ao se estudar cada componente isoladamente" (p. 60). ${ }^{8}$ A diferença de McWhinney em relação a Neighbour, é que McWhinney tem uma abordagem fenomenológica na qual admite o transcendente e/ou espiritual, ao se apoiar tanto em Whitehead quanto em E.F. Schumacher, enquanto Neighbour se apoia na física quântica para sustentar suas ideias, seguindo o pragmatismo britânico.

lan McWhinney e Roger Neighbour também utilizam a epistemologia de Thomas Kuhn ${ }^{10}$ para propor um paradigma diferente à medicina. Todavia, surgem respostas distintas e complementares que foram categorizadas separadamente para melhor compreensão. Tomando como referência a dimensão filosófica da MFC, os temas selecionados foram: (a) Paradigma organísmico de McWhinney e paradigma quântico de Neighbour; (b) Distinção entre a prática generalista e a prática do especialista focal; e (c) Relação médico-paciente e consigo mesmo. 


\section{Paradigma Organísmico de McWhinney e Paradigma Quântico de Neighbour}

Ambos autores constroem seu paradigma em oposição ao chamado modelo biomédico. McWhinney utiliza a teoria Kuhniana para evidenciar que esse modelo não consegue explicar algumas das "anomalias" encontradas na prática da MFC: (1) Dissociação adoecimento/doença: a maioria das pessoas que se sente mal não tem uma doença classificável, por exemplo, a forma como a pessoa experiencia a doença é trazida em consulta muitas vezes por sintomas clinicamente inexplicáveis (SCl); (2) Etiologia específica: nem sempre se estabelece o agente causal, como a imposição de tratar todos os pacientes com H. pylori positivo; (3) Separação entre mente e corpo, tratando somente as causas orgânicas, entretanto, a manifestação do adoecimento é tridimensional (biopsicossocial); e (4) Efeito curativo do placebo: abundantemente documentado nos ensaios clínicos (média de 35\% - variando de 10 a 90\%). ${ }^{1}$ Essas anomalias desafiam a ideia implícita e inscrita no paradigma da biomedicina de que as doenças são objetos com existência autônoma, traduzíveis pela ocorrência de lesões decorrentes de uma série de eventos desencadeados a partir de uma ou múltiplas causas. Assim, McWhinney propõe um paradigma organísmico (Quadro 1).

Quadro 1. Características do pensamento organísmico de McWhinney. ${ }^{111,12}$

1. Os organismos humanos têm as seguintes propriedades: crescimento, regeneração, cura, aprendizado, autoorganização e autotranscedência.

2. A terapia geralmente consiste em remover os obstáculos para a cura, sejam eles psicológicos ou físicos. A intervenção depende dos poderes de cura do organismo.

3. Os regimes tradicionais (e.g. das culturas populares e medicinas tradicionais) - nutrição equilibrada, descanso, sono sadio, exercícios, alívio da dor, apoio pessoal e paz mental - estimulam os poderes curativos naturais do organismo.

4. Um organismo reage aos traumas da vida como um todo. Toda doença significativa afeta o organismo em todos os níveis, desde o molecular até o cognitivo-afetivo.

5. Os sistemas auto-organizados, tais como os organismos, são multiníveis. As múltiplas vias de retroalimentação entre o paciente e o meio-ambiente e entre todos os níveis do organismo do paciente, exigem que pensemos em redes causais multidirecionais e não de modo linear.

6. O pensamento organísmico implica na completude entre mente e corpo e não na dualidade.

Roger Neighbour utiliza a definição do paradigma de Kuhn para comparar a física clássica de Newton com a física quântica de Einstein. Faz um paralelo entre a abordagem cientificista do especialista focal com o paradigma Newtoniano e do médico de família com o paradigma quântico. Desse modo, Neighbour (p. $99-100)^{8}$ propõe um paradigma quântico para a medicina (Quadro 2).

Apesar de utilizarem o mesmo arcabouço teórico, McWhinney tem um pensamento mais próximo dos sistemas auto-organizados e Neighbour mais próximo da física quântica e da indeterminação dos fenômenos. A ideia da matrioska é exatamente mostrar que uma estrutura impõe características na outra, assim como, um paradigma opera em outro. O novo paradigma não necessariamente exclui o velho, mas sim amplia o olhar sobre os fenômenos antes não explicados pelo antigo paradigma. 
Quadro 2. Características do pensamento quântico de Neighbour. ${ }^{8}$

\begin{tabular}{|l|l|}
\hline Paradigma quântico & Olhar contemporâneo do MFC \\
\hline $\begin{array}{l}\text { Existe somente uma coisa absoluta - a velocidade da } \\
\text { luz no vácuo. Como todas as outras coisas aparentam, } \\
\text { depende de onde estamos observando. }\end{array}$ & $\begin{array}{l}\text { Tudo que o médico lida - doença, adoecimento, saúde, } \\
\text { cura, consolo e especialmente a morte - pode ser } \\
\text { compreendido e manejado de várias formas e não } \\
\text { apenas de um modo puramente científico. }\end{array}$ \\
\hline $\begin{array}{l}\text { Mesmo das partículas fundamentais, tais como os } \\
\text { quarks, podem surgir uma variedade de sabores, } \\
\text { charme e estranhezas. }\end{array}$ & $\begin{array}{l}\text { Os indivíduos diferem entre si, não somente } \\
\text { fisicamente, mas também em seu funcionamento, } \\
\text { pensamento e comportamento. Variações individuais } \\
\text { são clinicamente significantes. }\end{array}$ \\
\hline Tempo é uma dimensão diferente em si própria. & $\begin{array}{l}\text { Para o paciente, um adoecimento não é um evento } \\
\text { isolado, mas um episódio em uma estória longa e } \\
\text { contínua. }\end{array}$ \\
\hline $\begin{array}{l}\text { Sempre existem elementos incontornáveis de incerteza } \\
\text { naquilo que tomamos como "conhecimento" e que não } \\
\text { podem ser resolvidos, não importa o quanto nos esforcemos. }\end{array}$ & $\begin{array}{l}\text { Mesmo o diagnóstico e o prognóstico mais abrangente } \\
\text { está envolto em incertezas que devem ser manejadas } \\
\text { em si mesmas. }\end{array}$ \\
\hline $\begin{array}{l}\text { Os eventos, às vezes, precisam ser descritos em termos } \\
\text { mais de probabilidades do que de certezas. }\end{array}$ & $\begin{array}{l}\text { A análise racional sistemática não é o único modo de } \\
\text { raciocínio diagnóstico. Às vezes, atalhos e "achismos } \\
\text { educados" são os únicos possíveis. }\end{array}$ \\
\hline $\begin{array}{l}\text { A causalidade nem sempre é linear e local. Eventos } \\
\text { remotos no tempo e no espaço podem ter efeitos não } \\
\text { previstos no que está acontecendo agora. }\end{array}$ & $\begin{array}{l}\text { As causas dos problemas médicos são usualmente } \\
\text { mais complexas e seus efeitos muito mais abrangentes } \\
\text { do que se pode perfeitamente apontar. }\end{array}$ \\
\hline $\begin{array}{l}\text { É possível estar em mais de um estado do ser ao mesmo } \\
\text { tempo - até que alguém venha verificar. }\end{array}$ & $\begin{array}{l}\text { O ato de consultar um médico restringe o paciente a um } \\
\text { papel limitado e fecha outras opções. Por exemplo, uma } \\
\text { pessoa assintomática com hipertensão não diagnosticada } \\
\text { é como o "gato de Schrödinger", ambos saudáveis e } \\
\text { doentes, até que alguém verifique a sua pressão arterial. }\end{array}$ \\
\hline
\end{tabular}

\section{Distinção entre a prática generalista e a prática do especialista focal}

McWhinney e Neighbour trazem em seus capítulos iniciais uma revisão histórica evidenciando como o pensamento médico foi se afastando da pessoa e se tornando cada vez mais impessoal. Isso ocorreu, segundo o ponto de vista dos autores, devido à valorização da especialização e da tecnologia.

McWhinney (p. 25-28) ${ }^{1}$ trabalha o tema do generalismo versus o especialismo de duas formas: a primeira descrevendo as nove características do médico de família (centrado na pessoa, foco no contexto, oportunidade para prevenção, nos riscos populacionais, pertencente a uma rede comunitária, idealmente que more na comunidade, etc.); a segunda (p. 37-38) didaticamente explicando a diferença entre o perfil generalista do especialista focal por meio de seis falácias: (1) O generalista tem que conhecer todo o campo do conhecimento médico; (2) Em qualquer campo da medicina o especialista sempre sabe mais do que o generalista; (3) Ao se especializar, a incerteza pode ser eliminada; (4) É apenas por meio da especialização que se pode atingir a profundidade do conhecimento; (5) À medida que a ciência avança, a carga de informação aumenta; e (6) O erro na medicina é geralmente causado por erro de informação. É possível notar a sistematização do trabalho de McWhinney e o cuidado em abordar todos os tópicos pertinentes à distinção entre o especialista focal e o MFC. 
Neighbour vai quase na contramão de McWhinney afirmando que a distinção entre "o especialismo e o generalismo é uma questão de estrutura mental (mindset), o modo de como fazer medicina se distingue não em termos de quem faz o que e onde, mas de como você pensa a sua prática médica" (p. 82). ${ }^{8}$ Mais à frente, Neighbour (p. 87) $)^{8}$ reforça a mesma ideia:

"A diferença importante entre [especialistas e generalistas] não reside no tipo de paciente ou doença com que trabalham, nem se trabalham dentro ou fora do hospital. A distinção, eu proponho, é mais de ordem cognitiva - do que ocorre em suas mentes, uma questão de como eles pensam a respeito do trabalho que desempenham. Existe uma maneira distinta do pensamento especialista e do generalista sobre a medicina e como ela deve ser praticada."

Esse modo de pensar pode ser compreendido como a analogia que Neighbour cria para diferenciar o olhar dos especialistas e dos generalistas. Os primeiros observam com lentes fotográficas fixas aos detalhes (close up), como uma lente de aumento, focam na queixa. Porém, ao fixarem o olhar sobre um ponto específico, perdem o contexto. Ele faz o contraste com os médicos que trabalham com saúde pública, os sanitaristas. Estes possuem lentes grande angular, que têm uma visão panorâmica, não perturbada pelos detalhes. Em contraste, a lente do generalista teria um zoom ajustável que permite ampliar e ver o contexto, focar e ver o detalhe, mas também realizar as interconexões entre indivíduo e contexto. As lentes dos generalistas teriam duas propriedades: (a) Ângulo variável; e (b) Direcionalidade: podendo olhar para fora, mas também girar sobre o seu eixo, e, inclusive, olhar para dentro de si mesmo. Assim, o próprio médico passa a ser o "foco" de reflexão.

\section{Relação médico-paciente e consigo mesmo}

Para ambos os autores, a relação com o paciente é muito significativa. Tanto McWhinney como Neighbour se dedicaram ao tema relacionado às habilidades de comunicação clínica. Eles reconhecem que o modelo biomédico resultou na precarização da relação médico-paciente, afastou o médico da pessoa/ paciente e da comunidade, inclusive até dele mesmo como pessoa, médico e cidadão. Para McWhinney (p. 22), ${ }^{1}$ existe uma "negligência da educação médica quanto ao desenvolvimento emocional dos médicos". As escolas médicas valorizam as qualidades técnicas e saberes teóricos e não apreciam as características humanas, éticas e comunicativas dos médicos em formação.

Entretanto, vários estudos comportamentais começaram a demonstrar a importância da experiência do adoecimento (illness) e dos fatores comportamentais e sociais envolvidos nas causas das doenças. Desse modo, a MFC começou a perceber o papel central do médico nos processos terapêuticos. De acordo com McWhinney (p. 47): ${ }^{1}$

"Saber escutar com habilidade e ser sensível às pistas sutis por meio das quais se expressam as necessidades emocionais. Também é necessário ter autoconhecimento, pois o médico deve estar consciente da forma como os seus próprios sentimentos e experiências familiares o afetam."

Para entender os aspectos subjetivos da pessoa, o MFC precisa conhecer a si próprio e suas emoções. O mergulho no outro expande nosso ser, e, ao mesmo tempo, perturba e agita nossa consciência. Confrontar os sentimentos e sofrimentos dos pacientes exige muito dos MFCs e mobiliza a estrutura interna da pessoa, isto é, o nosso paciente interno. O autoconhecimento e o entendimento de nossa própria contratransferência são importantes. Para McWhinney (p. 132), ${ }^{1}$ somente "podemos entender os outros na medida em que conhecemos a nós mesmos." 
Roger Neighbour também traz a questão do autoconhecimento como base para uma prática médica diferenciada. Para tanto, o MFC necessita olhar para o que denominou "the inner physician", o leigo dentro de nós, o legado psicoafetivo anterior a sua formação médica, que inclui: "pensamentos, emoções, memórias, motivações, atitudes, hábitos, princípios e crenças que trazemos, que permeia e que segue para além da escola médica (p. 27)". ${ }^{1}$ Para o autor, esse aspecto constitucional é desvalorizado pela medicina. Neighbour (p. 28) ${ }^{8}$ afirma que:

"A qualidade e a efetividade da prática dos médicos são afetadas pelo grau de intimidade e o quão confortáveis eles se sentem em relação ao seu próprio mundo interior, bem como, o quanto permitem que a sua humanidade singular seja um fator a influenciar seu trabalho clínico."

O autor conclui que os especialistas focais tendem a anular seu "inner physician" e costumam separar seus aspectos psicológicos da prática médica. Os generalistas, por outro lado, costumam ter maior familiaridade com seu mundo interior, entendendo esse aspecto como "vantajoso e que adiciona valor, poder e insight no seu papel terapêutico" (p. 28). ${ }^{8} \mathrm{O}$ autor então designa o MFC como "doutor-enquanto-pessoa" e o especialista focal "doutor-enquanto-especialista". Neighbour conclui que "os médicos que excluem seu 'inner physician' tendem a pensar e a praticar a medicina ao modo do especialista focal [...] e aqueles que permitem um certo grau de envolvimento no modo como pensam e praticam a medicina tendem a ser generalistas" (p. 31). ${ }^{8} \mathrm{O}$ "inner physician" seria uma bússola para navegar nas incertezas da prática generalista e quase um pré-requisito para estabelecer uma relação médico-paciente terapeuticamente efetiva. Para Neighbour (p. 25), ${ }^{8}$ a base da empatia encontra-se em "conhecer nossas próprias vulnerabilidades, visto que elas nos ensinam a respeitar as dos outros". O amador dentro de nós, que antecede a formação médica, é quem retém a capacidade de compaixão, essencial para o desenvolvimento da empatia e o trabalho assistencial. Portanto, tanto McWhinney como Neighbour reconhecem que a pedra angular e, talvez, o segredo de uma prática médica efetiva esteja no desenvolvimento das qualidades do mundo interior de cada médico.

Essa dimensão interior tem importância devido ao efeito do observador. A medicina do século XIX afasta o observador (médico), assim como a física de Newton propunha a separação entre o observador e o objeto. Já a física quântica do século XX coloca a consciência do observador como parte essencial. Nesta última, o observador precisa ter o conhecimento do todo. Para McWhinney (p. 135)1 "o médico não é apenas o observador da pessoa, mas um meta-observador do ser da pessoa como um todo." Na mesma linha, Neighbour (p. 100) ${ }^{8}$ considera o "inner physcian" como parte integral da consulta e "o fluxo consciente e lúcido do observador-médico do seu mundo interior é essencial para construir uma compreensão abrangente do mundo exterior clínico".

\section{CONCLUSÃO}

A medicina de família e comunidade requer uma constante problematização dos seus pressupostos, visto que seu foco não se restringe aos aspectos biológicos do ser humano. A leitura de Neighbour em relação à McWhinney confirma a necessidade de pensar a prática e a teoria da MFC. A formação e a expansão da MFC, tanto nos cursos de graduação quanto nos programas de residência, necessitam utilizar a epistemologia da MFC para valorizar e estimular o "inner physician" que existe em cada um de nós. 


\section{Contribuição dos autores}

BRJ e AHN contribuíram igualmente na elaboração do artigo desde sua concepção, metodologia e análise dos textos selecionados, sua redação e revisão. Os autores declaram não haver conflito de interesse na elaboração do manuscrito e que não houve nenhum financiamento nesse processo.

\section{REFERÊNCIAS}

1. McWhinney IR, Freeman T. Manual de Medicina de Família e Comunidade. 3aㅡ ed. Porto Alegre: ARTMED; 2010.

2. Starfield B, Shi L, Macinko J. Contribution of primary care to health systems and health. Milbank Q. 2005;83(3):457-502. DOI: https://doi. org/10.1111/j.1468-0009.2005.00409.x

3. Franks P, Clancy CM, Nutting PA. Gatekeeping revisited — protecting patients from overtreatment. N Engl J Med. 1992;327(6):424-9. DOI: https://doi.org/10.1056/NEJM199208063270613

4. Norman AH, Tesser CD. Prevenção quaternária: as bases para sua operacionalização na relação médico-paciente. Rev Bras Med Fam Comunidade. 2015;10(35):1-10. DOI: https://doi.org/10.5712/rbmfc10(35)1011

5. Pimlott N. Reflecting on Dr. Ian McWhinney. Can Fam Physician [Internet]. 2012; [2019/ fevereiro/ 15]; 58(11):1187-8. Disponível em: http://www.ncbi.nlm.nih.gov/pubmed/23152451

6. Neighbour R. The Inner Consultation: how to develop an effective and intuitive consulting style. Abingdon, UK: Radcliffe Publishing; 2005.

7. Neighbour R. The inner apprentice: an awareness-central approach to vocational training for general practice. Abingdon, UK: Radcliffe Publishing; 1992.

8. Neighbour R. The Inner Physician: Why and How to Practise "Big Picture Medicine." London, UK: Royal College of General Practitioners; 2016.

9. McWhinney IR. A Textbook of Family Medicine. $2^{\text {nd }}$ ed. New York: Oxford University Press; 1997.

10. Kuhn TS. The structure of scientific revolutions. $2^{\text {nd }}$ ed. Chicago: University of Chicago Press; 1970.

11. McWhinney IR. Being a general practitioner: what it means. Eur J Gen Pract.2000;6(4):135-9. DOI: https://doi.org/10.3109/13814780009094320

12. McWhinney IR. Why we need a new clinical method. Scand J Prim Health Care [Internet]. 1993 Mar [2019/ fevereiro/ 15]; $11(1): 3-7$. Disponível em: http://www.ncbi.nlm.nih.gov/pubmed/8484077 